\title{
Research on Flight Numerical Simulation of Aerodynamic Conditions
}

\author{
Ze Yang ${ }^{1 *}$, Chaoyi Wang ${ }^{1}$ and Junjie Zhou ${ }^{1}$ \\ ${ }^{1}$ Unit95926, Jilin University, Jilin University, Jilin Changchun 130022, China. \\ *yuyangtao312@163.com \\ * The corresponding author
}

Keywords: Simulation technology; Aerodynamic parameters; Dynamic Characteristics; N-S equation

\begin{abstract}
In recent years, due to the development of science and technology, the use of computer systems to simulate flight exercises, as well as in the digital world or the use of digital and reality in combination with the completion of flight exercises has become possible. Moreover, with the further development of technology, the application of this simulation technology will be more extensive, and the effect of simulation will be more and more highly.

Modern aircraft control is particularly complex, especially the new fighter, with ultra conventional motor performance, intelligent avionics systems and electronic warfare systems, as well as powerful fire control system. Pilot not only to have superb driving skills, should also have skilled in the use of other systems to deal with and special ability, which to a pilot proposed higher requirements, improve the level of training pilots to become air force of each country need to solve problems. Therefore, all kinds of flight simulation system have become one of the important means to train pilots. Aircraft under different aerodynamic parameters, wings, tail, engine inlet leading edge, windshield, airspeed tube, antenna with different gas dynamic characteristics.

In this paper, the typical aerodynamic conditions are obtained by numerical simulation. The numerical simulation method is used to study the influence of aerodynamic characteristics on the performance of the wing. Main research work are:

The N-S equation to calculate the icing of air flow field, turbulence model selected S-A model suitable for the research aircraft, with Euler method to establish air control equation;

The flight simulation system is built by using the distributed simulation technology, and the flight simulation framework based on the high level architecture is preliminarily constructed.
\end{abstract}

\section{Flight Motion Description}

According to the requirement of the design and Simulation of the flight envelope protection control law, the nonlinear flight dynamics model of six degree of freedom is used in this paper. Based on the flight dynamics and control toolbox FDC(Flight Dynamics and Control) of MATLAB, the aircraft flight dynamics model is established. The model considers the sensor noise, nonlinear flight dynamics, data filtering, hinge moment modeling, atmospheric disturbance model and other factors. According to different research objects and purposes, the corresponding linear or nonlinear flight dynamics models should be established in the study of the flight dynamics characteristics of the aircraft in the aerodynamic environment. The general research of flight quality and the system parameter identification of the aircraft can be considered using the linearized equation. Aircraft airworthiness analysis often involves critical flight conditions (such as tail plane stall characteristics analysis) and the nonlinear effect of the state was, we must consider the establishment of corresponding nonlinear dynamics model. Sometimes, in order to calculate and shorten the calculation time, and take into account the actual situation of the original data of the aerodynamic force, although the linear equation is not small, but still use the vertical and horizontal decoupling of the aircraft motion equation. 


\section{Aerodynamic Characteristics Analysis}

Under different aerodynamic parameters, the numerical analog method of aircraft gas dynamic characteristics is a prerequisite for the calculation of air flow field. In this paper combined with the characteristics of air flow under the aircraft icing conditions. We first discuss the calculation method of air flow field numerical simulation of aircraft flight.

Foreign gas dynamic flow field research and development prediction software in most of the potential flow solution or the Euler equation to calculate the air flow field, because this approach does not consider the viscosity of air, unable to deal with flow separation, therefore in the integral boundary layer method is used to solve the heat transfer coefficient of need and the flow separation region the contour smoothing processing, this method when serious flow separation and processing will have great deviation. Under severe weather conditions, the effect of viscous flow on the surface of the wing can not be ignored, and the method can not accurately describe the flow field when the serious air flow is separated in flight. So it has a great advantage to solve the air flow field with the N-S equation in the numerical simulation of the flight. In the establishment of $\mathrm{N}-\mathrm{S}$ equation, the first to do the following basic assumptions:

Fluid is continuous.

The fields involved are differentiable, such as pressure, density, velocity, etc.. N-S partial differential equations are established to represent the mass conservation, conservation of momentum and conservation of capacity of the flow field. The N-S equations expressed by the stress tensor are as follows:

Continuous equation:

$$
\frac{\partial \rho}{\partial \mathrm{t}}+\frac{\partial \rho \mathrm{u}_{\mathrm{j}}}{\partial \mathrm{x}_{\mathrm{j}}}=0
$$

Momentum equation:

$$
\frac{\partial}{\partial \mathrm{t}}\left(\rho \mathrm{u}_{\mathrm{i}}\right)+\frac{\partial}{\partial \mathrm{x}_{\mathrm{j}}}\left(\rho \mathrm{u}_{\mathrm{i}} \mathrm{u}_{\mathrm{j}}\right)=\frac{\partial}{\partial \mathrm{x}_{\mathrm{j}}}\left(\tau_{\mathrm{ij}}\right)
$$

Energy equation:

$$
\frac{\partial(\rho \mathrm{H}-\mathrm{p})}{\partial \mathrm{t}}+\frac{\partial\left(\rho \mathrm{u}_{\mathrm{j}} \mathrm{H}\right)}{\partial \mathrm{x}_{\mathrm{j}}}=\frac{\partial}{\partial \mathrm{x}_{\mathrm{j}}}\left(\mathrm{u}_{\mathrm{i}} \tau_{\mathrm{ij}}\right)-\frac{\partial}{\partial \mathrm{x}_{\mathrm{j}}}\left(\frac{\mu}{\operatorname{Pr}} \frac{\partial \mathrm{h}}{\partial \mathrm{x}_{\mathrm{j}}}\right)
$$

The Reynolds stress and energy equation in the momentum equation are not closed to the equations, and the corresponding turbulence models are needed to be established. Turbulence is one of the most complicated theoretical problems in fluid mechanics, which is a kind of nonlinear fluid motion in space and time disorder. A large number of turbulence models have been established so far, but there is not a general turbulent model. Aircraft wing icing will produce the ice shape complex, often accompanied by the flow separation, also ice surface roughness influence on the flow and heat transfer is very large, so the turbulent model selection S-A turbulence model. S-A model can reasonably simulate the small separated turbulent flows and is the one equation model, compared with the two equation model, the amount of calculation is small, is considered to be one of the most successful one equation and two equation model of turbulence model. The governing equation of the S-A model is a partial differential equation of the variable associated with the viscosity of the fluid:

$$
\frac{\partial}{\partial \mathrm{t}}(\widetilde{\rho \mathbf{v}})+\frac{\partial}{\partial \mathrm{x}_{\mathrm{j}}}\left(\widetilde{\rho \mathbf{u}}_{\mathrm{j}} \tilde{\mathrm{v}}\right)=\mathrm{C}_{\mathrm{b} 1} \tilde{\mathrm{S}} \rho \widetilde{\mathrm{v}}+\frac{1}{\sigma}\left[\nabla \cdot((v+\tilde{v}) \nabla \widetilde{\rho \mathbf{v}})+\mathrm{C}_{\mathrm{b} 2}(\sqrt{\bar{\rho} \widetilde{\mathbf{v}}})^{2}\right]-\mathrm{C}_{\mathrm{w} 1} \mathrm{f}_{\mathrm{w}} \bar{\rho} \rho\left(\frac{\widetilde{\mathbf{v}}}{\mathrm{d}}\right)^{2}
$$


In summary, the above N-S equations are solved to determine the disturbed flow field information of the aircraft, and the basic steps of the method of semi implicit solution to the equation group of the pressure coupled equations are solved:

According to the classical hydrodynamic pressure field values obtained;

Solving the equations of the velocity extremum $\mathrm{U}^{*} 、 \mathrm{~V}^{*}$;

Solving the pressure equation $\mathrm{P}^{*}$;

Additional offset based on $\mathrm{P}^{*}$ value, get $\mathrm{P}$;

According to $\mathrm{U}^{*}, \mathrm{~V}^{*}$ and $\mathrm{P}^{*}$ for $\mathrm{U}, \mathrm{V}, \mathrm{W}$;

If the numerical value of the flow field is affected by the convection term or source term, the discrete equation of the flux of the flow field is required. If there is no effect in the flow flux convergence after solving the equations;

The calculated pressure $\mathrm{P}$, substitution for the extreme value of the solving process, repeated throughout the settlement process until according to the setting of the convergence criteria that a suitable solution.

In summary, the flow field around the airfoil can be obtained by solving the discrete equations by solving the algebraic equations, as shown Fig.1.

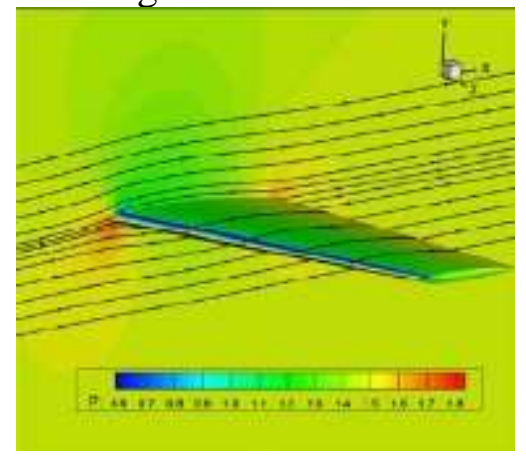

Figure. 1 The flow field around the airfoil

\section{Simulation Architecture}

The application of HLA as a soft bus, acts as the core of the collaborative simulation architecture, combining the pneumatic simulation analysis software with the logic analysis software to realize the seamless connection. HLA uses the object oriented design concept to construct the collaborative simulation system. This kind of simulation system is oriented to the simulation object planning and simulation system. The research on the relationship between aerodynamic analysis and control characteristic is a complex simulation system, which is broken down into a small union according to the functional requirements. According to their own needs, the small federal application field mature commercial simulation software to complete the simulation analysis. These results of the analysis of the federal members, in the form of a unified output data, through the RTI bus to achieve information exchange, improve the degree of data reuse. The simulation architecture is shown in Fig.2. 


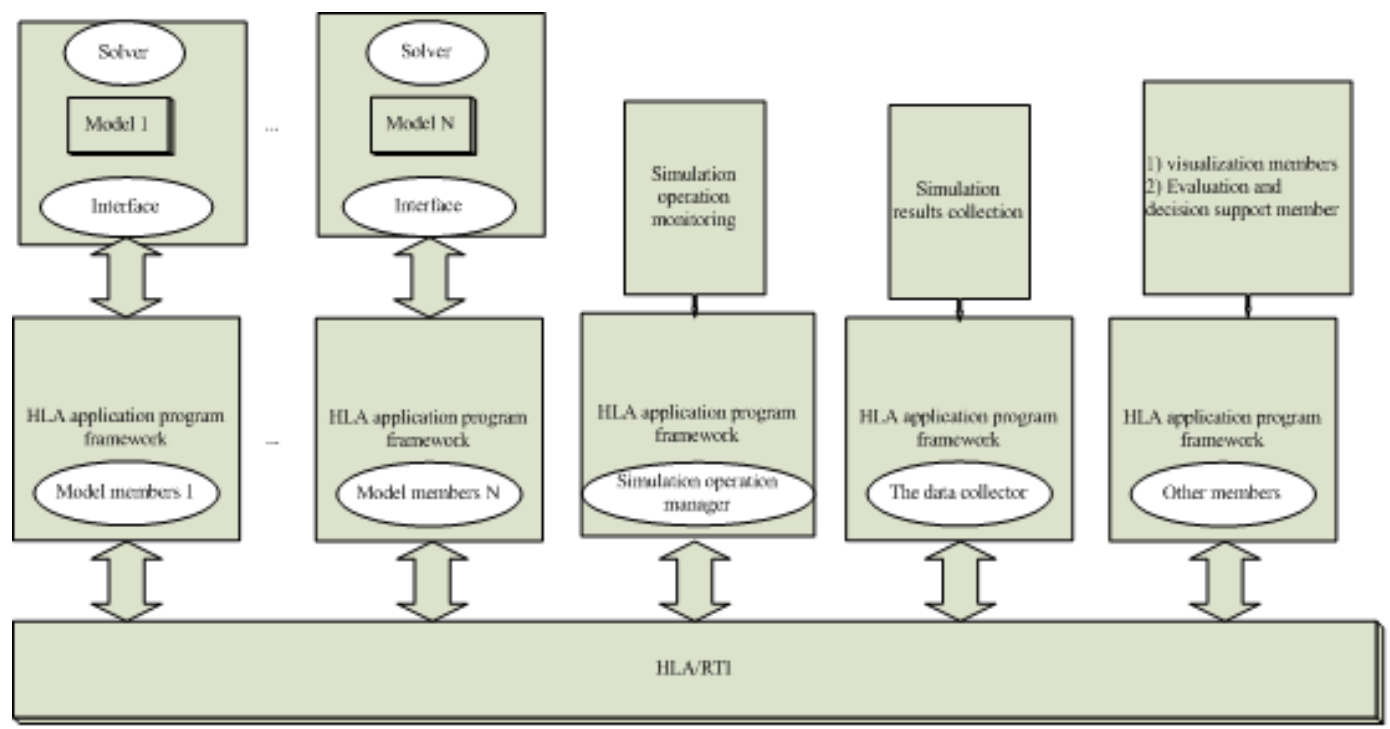

Figure. 2 Simulation architecture

\section{Conclusion}

This paper mainly introduces the three modules of aircraft motion numerical simulation: flow field calculation, aircraft motion trajectory calculation, collaborative simulation analysis. The N-S equationsand S-A turbulence model to calculate the air flow field, the Euler method is used to build aircraft motion equations and two-phase flow control equation, and the finite volume method through the numerical calculation of the equations and determine the aircraft trajectories. HLA is used to construct the simulation architecture, and the flight simulation analysis under complex weather conditions is completed. The results show that the method can effectively simulate the flight of the aircraft. Can be used to identify the main aerodynamic parameters of aircraft flying, analysis of its aerodynamic characteristics.

\section{References}

[1] Tao Bo, combat behavior modeling technology of [J]. of national defense science and Technology University, 2002: 1.

[2] Wang Wenguang, Liu Xichun, Wang Weiping, et al. Research progress on the high level architecture of service oriented [J]. computer integrated manufacturing system, 2008, 14 (9): 1665 1675.

[3] JONES R. XML Provides Extra Help for Embedded Systems[J]. Computing and Control Engineering. 2005, 16(6): 42-47.

[4] Liu Xing, flight principle [M]. Science Press, 2011:176-185. 